\title{
Delineation of Lung Tumor with Neural Networks
}

\author{
Kalpana R. A ${ }^{\mathrm{a}}$, Ganga A ${ }^{\mathrm{b}}$, Deepika R ${ }^{\mathrm{b}}$, Kiruthiga Devi M ${ }^{\mathrm{b}}$, Ramya $\mathrm{N}^{\mathrm{b}}$ \\ ${ }^{a l, b}$ Assistant Professor, Dept of CSE, Sairam Engineering College Chennai, India
}

\begin{abstract}
Due to the picture similar region of interest, local area, and the effect of respiration, separating a lung tumor from neighboring tissue from a collection of magnetic resonance images (MRI) faces a number of challenges. However, precise tumor segmentation is needed when preparing radiation therapy in order to prevent excessive radiation exposure to healthy tissues. Delineation of the whole MRI pattern by hand is boring, time-consuming, and costly. Using neural networks, this research studies the automated tracking of tumor borders during radiation therapy. To improve the accuracy of lung tests, we proposed using neural network architecture with fuzzy clustering.
\end{abstract}

Keywords: Lung Tumor Detection, Neural Network, Lung Regions, Fuzzy Clustering.

\section{Introduction}

Lung cancer is a condition that causes cells to divide in the lungs uncontrollably. This causes the growth of tumors that reduce a person's ability to breathe. Identifying lung cancer in its earliest stages can be difficult, however, because the symptoms may be similar to those of a respiratory infection, or there may be no symptoms at all. Recently, image processing techniques are widely used in several medical areas for image improvement in earlier detection and treatment stages. Lung cancer is a disease of abnormal cells multiplying and growing into a tumor. Image segmentation is a fundamental step in computerized image analysis. It deals with separating classes in an image into continuous and separate regions. Delineation of lung tumor from adjacent tissue from a series of magnetic resonance images (MRI) poses various problems due to the image identical region of interest, surrounding area and the influence of respiration. This study investigates automatic tracking of tumor boundaries during radiation therapy using neural networks. We proposed to use a neural network architecture with fuzzy clustering to improve the accuracy in lung studies.

\footnotetext{
${ }^{1}$ Kalpana R A, Assistant Professor, Dept of CSE, Sairam Engineering College, Chennai, India. Email:kalpana.cse@sairam.edu.in ${ }^{1}$
} 


\section{Related Works}

In this modern world, all the people were busy with their tight schedule. Due to which most of the people were not health conscious and suffer from various diseases. One of the commonly occurring defects among human is lung failure which occurs due to various reasons.[1] Over the last decade, a plethora of computer-aided diagnosis (CAD) systems have been proposed aiming to improve the accuracy of the physicians in the diagnosis of interstitial lung diseases (ILD). The feature extraction method relies on local spectral analysis using a DCT-based filter bank. After convolving the image with the filter bank, q-quantiles are computed for describing the distribution of local frequencies that characterize image texture. Then, the gray-level histogram values of the original image are added forming the final feature vector. The classification of the already described patches is done by a random forest (RF) classifier.[2] Current computed tomography (CT) technology allows for near isotropic, sub millimeter resolution acquisition of the complete chest in a single breath hold. These thin-slice chest scans have become indispensable in thoracic radiology, but have also substantially increased the data load for radiologists. Automating the analysis of such data is, therefore, a necessity and this has created a rapidly developing research area in medical imaging.[3] Diagnosis of lung nodules and cancers is a critical and urgent problem in clinical diagnosis. This thesis is to design and build a computer aided lung ground glass opacity (GGO) nodules and large lung cancers diagnosis system which aims to quantify the volumetric change of the lung GGO nodulesandlarge lung cancers between the pretreatment and post-treatment. Sputum cytology examination has been shown in several studies to lead to detection of lung cancer at an earlier stage, resulting in an improved 5-yearsurvival rate.

\section{Existing System}

In the existing system, lung features are extracted by manual analysis and it is very time consuming process. The thresholding method is not accurate in comparing the normal and abnormal lung.

- Not applicable for multiple images for Tumor detection in a short time

- Difficult to get accurate results

- Medical Resonance images contain a noise caused by operator performance which can lead to serious inaccuracies classification

\section{Proposed System}

In proposed methodology, the MRI (Magnetic Resonance Image) image is preprocessed using DWT (Discrete Wavelet Transform). Key features are extracted using Gray level co-occurrence matrix (GLCM).A data set oftest data containing normal and abnormal lung MRI images are classified using Neural Network. Feature extraction using GLCM Features. In the similar way, identifies contours by identifying differences between regions (edges).

The input test image is pre-processed using Discrete Wavelet Transform. Image enhancement or pre-processing is done to remove noise and brighten the image to identify the key features. The Gray level co-occurrence matrix determines the texture 
features of an image by calculating how often the pixel pairs with specific values. First, it creates a GLCM and then extracts the significant texture features from this matrix. Training dataset consists of a set of images consisting of normal and abnormal lung. The training process is same as the processing of test image, which involves preprocessing, and feature extraction. The features extracted from the trained set are used for comparison with the features extracted from the test image for accurate classification of lung tumor, which is done using method of neural network. Fuzzy clustering is an unsupervised clustering technique which does not demand a human interaction to decide the clustering criteria. It performs on distance metrics between the data point and cluster centre by assigning a membership function to each data point corresponding to each cluster centre. Comparing to k-means clustering fuzzy-c means works well for overlapped data set thus enabling us to find the accurate location of the lung tumour.

\section{Working System}

A software application in general is implemented after navigating the complete life cycle method of a project. System implementation is an important stage of theoretical design is turned into practical system. Implementation is the stage of the project when the theoretical design is turned out into a working system. Thus it can be considered to be the most critical stage in achieving a successful new system and in giving the user, confidence that the new system will work and be effective. The implementation stage involves careful planning, investigation of the existing system and its constraints on implementation, designing of methods to achieve changeover and evaluation of changeover methods. Each program is tested individually at the time of development using the data and has verified that this program linked together in the way specified in the programs specification, the computer system and its environment is tested to the satisfaction of the user. The system that has been developed is accepted and proved to be satisfactory for the user and so the system is going to be implemented very soon. A simple operating procedure is included so that the user can understand the different functions clearly and quickly. The final stage is to document the entire system which provides components and the operating procedures of the system.

\section{System Architecture And Implementation}

A modular design reduces complexity, facilities change (a critical aspect of software maintainability), and results in easier implementation. The five important criteria that enable us to evaluate a design method with respect to its ability to define an effective modular design are: Modular decomposability, Modular Comps ability, Modular Understandability, Modular continuity, Modular Protection.

\section{Module 1: Preprocessing and Feature extraction}

In this module resizing and removing the unwanted data from the blood microscopic image dataset and down sampling the images so that we can train faster. Preprocessing operations are applied on input data image for smoothening the cancer part. Dilation and erosion process will be used to enhance (smoothening) the cancer region by 
removing the unwanted pixels from outside region of cancer part. These morphological operations are performed on images based on shapes. The output pixel is determined by using these processing pixel neighbors. Feature extraction is a process of dimensionality reduction by which an initial set of raw data is reduced to more manageable groups for processing. A characteristic of these large image data sets is a large number of variables that require a lot of computing resources to process.

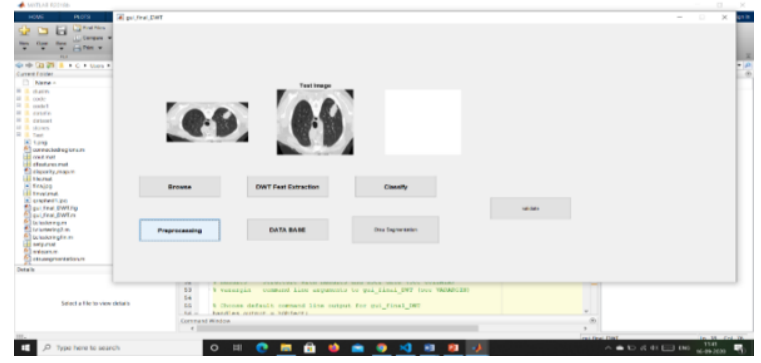

Figure 1. Preprocessing and Feature extraction

\section{Module 2: GLCM with Segmentation}

Given Input image composed of pixels each with an intensity (a specific gray level), the GLCM is a tabulation of how often different combinations of gray levels co-occur in an image or image section. $\mathrm{K}$-means clustering algorithm is an unsupervised algorithm and it is used to segment the interest area from the background. But before applying $\mathrm{K}$ means algorithm, first partial stretching enhancement is applied to the image to improve the quality of the image. Subtractive clustering method is data clustering method where it generates the centroid based on the potential value of the data points. So subtractive cluster is used to generate the initial centers and these centers are used in k-means algorithm for the segmentation of image.

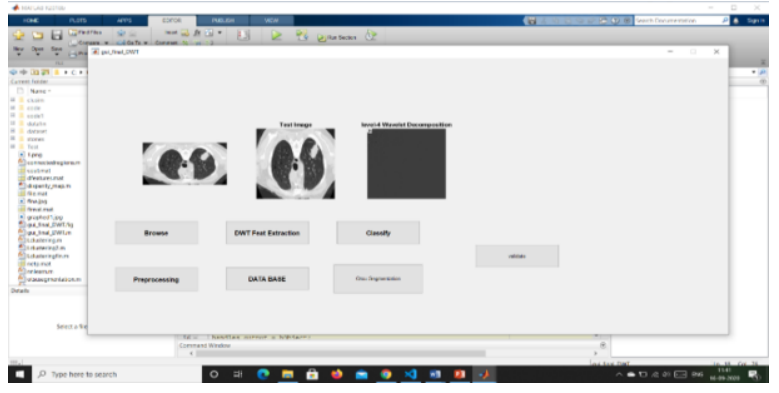

Figure 2. GLCM with Segmentation 


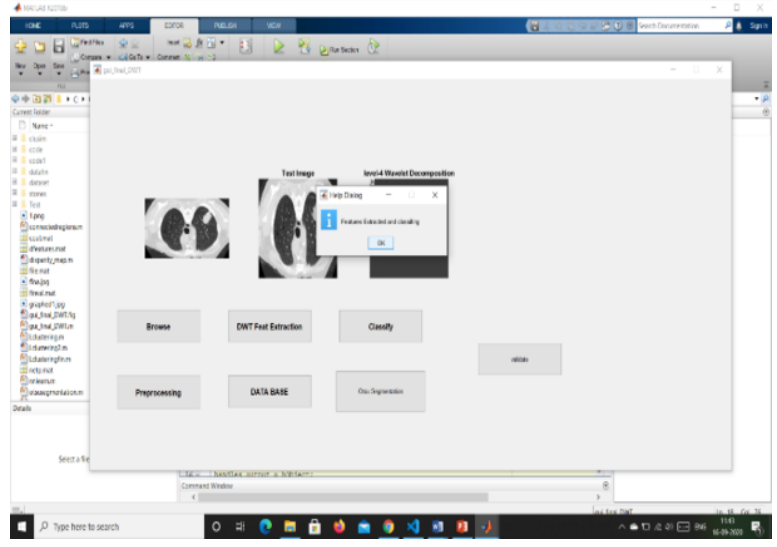

Figure 3. NN Training and Classification

\section{Future Scope}

In future work, the proposed methodology will be designed for real time implementation by interfacing it with the scanning machines. The captured lung image will be subjected to the proposed algorithm to identify the affected region and for accurate classification of lung tumor.

\section{Conclusion}

The project presented that automated CT lung cancer classification for early stage abnormality detection of Pneumonia and TB with use of neural network classifier and spotting of cancer was done with image segmentation. Pattern recognition was performed using Back propagation with feed forward neural network and pattern will be characterized with the help of principal component analysis. Spatial fuzzy clustering algorithm was utilized effectively for accurate cancer detection to measure the area of abnormal region. From an experiment, system proved that it provides better classification accuracy with various stages of test samples and it consumed less time for process.

\section{References}

[1] Dignam JJ, Huang L, Ries L, Reichman M, Mariotto A, Feuer E, Estimating cancer statistic and othercause mortality in clinical trial and population-based cancer registry cohorts, Cancer 10, Aug 2009.

[2] T. C. Kennedy, Y. Miller and S. Prindiville,Screening for Lung Cancer Revisited and the Role of Sputum Cytology and Fluorescence Bronchoscopy in a High-Risk Group, Chest Journal, vol. 10, pp. 72-79, 2005.

[3] Z. Daniele, H. Andrew, J. Nickerson,Nuclear Structure in Cancer Cells, Nature Reviews Cancer, Medical School, vol. 4, no. 9, pp. 677-87, USA, Sep. 2004.

[4] Georgiadis. Et all,Improving Lung Cancer characterization on CT by probabilistic neural networks and non-linear transformation of textural features, Computer Methods and program in biomedicine, vol 89, pp24-32, 2008. 